\title{
Cross-cultural contextualisation for recommender systems
}

\author{
Minsung Hong · Sojung An · Rajendra Akerkar · David Camacho Jason J. Jung
}

\begin{abstract}
Cultural Heritage $(\mathrm{CH})$ domain is rapidly moving from traditional heritage sites into smart cultural heritage environment through various technologies. As one of the important technologies in the smart space, Recommender Systems (RSs) have been widely utilised to personalised services and matching visitors' goals and behaviours. Whereas, cultural difference is often considered a barrier to technology transfer or adoption. However, few studies focus on how the cultural factor influences recommendation despite cultural difference largely affects user preferences in the RSs. Furthermore, existing researches have mainly analysed evaluation results of their recommendation to reveal cultural differences, rather than utilising the cross-cultural factors into RSs. In this paper, we propose a novel concept of cross-cultural contextualisation and a model to compute the cross-cultural factor affecting users (countries or cultures) preferences by using matrix factorisation and clustering techniques. In addition, we discuss how to apply the model to RSs in $\mathrm{CH}$ domain through cross-domain recommendation techniques. Note that the two computational techniques were used to analyse cross-cultural factors which impact to user preferences, rather than to recommend items. In other words, the proposed model and computing results capable of utilisation into the other RSs as well as various research fields. Results of experiments with a real-world dataset showed effectiveness of the proposed model and supported that there is cultural difference influencing users' rating behaviours. Furthermore, a systematic analysis of dataset and the experimental results presented that individual users could be considered as countrywise groups for the model to analyse the cross-cultural factors.
\end{abstract}

Keywords Cross-cultural contextualisation $\cdot$ Computational analysis $\cdot$ Recommender system $\cdot$ Matrix factorisation $\cdot$ Cultural analysis $\cdot$ Smart cultural heritage

\section{Introduction}

Nowadays the domain of Cultural Heritage $(\mathrm{CH})$, which represents a worldwide resource of inestimable value, attracting millions of visitors every year to monuments, museums, exhibitions and historical centres, has turned out to be a research field where different perspectives should be analysed (Chianese et al. 2016). Its fundamental aspects to be enhanced are the sustainable use by, promotion to and enjoyment for people and the tutelage of cultural objects and spaces for former purposes. Therefore, the $\mathrm{CH}$ domain can be considered places where people interact with objects capable of arousing interest and excitement. In order to efficiently and effectively foster such environments, many practitioners and researchers have tried to transform traditional heritage sites into smart cultural heritage environment. The transformation has been more promoted with the advance of various technologies such as Recommender Systems (RSs) (Hong et al. 2017; Pavlidis 2019), Internet of things 
(Chianese and Piccialli 2016), cloud services (Siountri et al. 2018), wireless sensor network and 5G (Chianese and Piccialli 2014; Wang et al. 2018), Virtual and Augmented Reality (VR/AR) (Jung et al. 2018; Bekele et al. 2018). According to Chianese et al. (2015), the smart cultural environments have complex relationships between $\mathrm{CH}$ and the new technologies, and RSs are one of important components in the smart space. Indeed, it is widely agreed that the purpose of Cultural Heritage exhibitions is rapidly moving from an old vision, that provides static information consisting of a large amount of cultural signs, to novel personalized services and matching the visitors' goals and behaviours by considering their preferences and context information (Bartolini et al. 2013).

On the other hand, according to Lee (2016) cultural difference is often considered a barrier to technology transfer, and Jung et al. (2018) supported the idea that information system is largely influenced by culture. Therefore, there are many researches to illuminate the phenomenon in RSs (Chen and Pu 2008; Tang et al. 2011; Berkovsky et al. 2018). Hofstede (1980) defined culture as "the collective programming of the mind which distinguishes the members of one human group from another". Furthermore, the author and his colleague suggested five cultural dimensions [long-term orientation was subsequently added by Hofstede and Bond (1988)] through which countries are comparable: masculinity/femininity; power distance; time orientation; individualism/collectivism; and uncertainty avoidance. Among these five dimensions, RSs have relations with collectivism and uncertainty avoidance. Collectivism can be considered as the recommendation functionality using collaborating filtering algorithm which utilises preferences of a group of people who have similar characteristics. Whereas, uncertainty avoidance is related with the purpose of RSs as people use the systems to reduce alternatives therefore to alleviate uncertainty in making purchase decision (Choi et al. 2014).

Although cultural difference has close relationship with RSs, few works focus on how the cultural factor influences recommendation (Choi et al. 2014; Chen and Pu 2014; Chu and Huang 2017). Even existing researches investigating the role of cultural factors in personalised systems have mainly focused on the analysis of evaluation results of their recommendation to reveal cultural differences, rather than the utilisation of cross-cultural factors, which influence users' rating behaviours, into the RSs. To the best of our knowledge, there is no study to apply cultural difference to recommendation for $\mathrm{CH}$ domain. Therefore, a computational method for the analysis and utilisation of cross-cultural factors to be applied into RSs is essential. It enables to foster studies enabling to enhance above purposes of $\mathrm{CH}$ domain that would obtain valuable benefits on utilising RSs.

In this paper, we discuss how to analyse cross-cultural factors by using computational techniques (e.g., matrix factorisation and clustering) and introduce two methodologies for applying the computational results to RSs in $\mathrm{CH}$ domain. Thus, our primary contribution is threefold.

- We define a novel concept of "Cross-cultural contextualisation" and present the problem definition for contextualising cultural differences in RSs.

- Regarding these definitions, we propose a contextualisation model for computing the cross-cultural factor, which influences user preferences in RSs, by using Matrix Factorisation (MF). Thus, the model capable of directly applying to other RSs also.

- We introduce and discuss two empirical methodologies based on cross-domain recommendation to utilise the computational model into RSs for $\mathrm{CH}$ objects. Thus, the two methodologies do not limited by domains because of high freedom of the cross-domain recommendation.

The rest of this paper is organised as follows. Section 2 reviews existing literature on the recommendation in $\mathrm{CH}$ domain and the cross-cultural analysis in RSs. In Sect. 3, we define an essential concept to compute cultural differences and propose a model based on matrix factorisation. Section 4 evaluates the model, and then we discuss two methodologies applying the computational model to RSs for $\mathrm{CH}$ domain in Sect. 5. Finally, Sect. 6 describes conclusion and future work.

\section{Related work}

\subsection{Cultural heritage recommendation}

During the last years, one key sector where recommendation facilities are playing a key role consists in the tourism market and the CH (Nguyen et al. 2017; Pavlidis 2019). Apart from the naive example of suggesting hotels, RSs are changing the way that people enjoy CH sites of historical and artistic interests (Su et al. 2019). In this regard, RSs are more and more playing an important role in our life, enabling users to find "what they need" within very large data collections and supporting various applications from e-commerce to $\mathrm{CH}$ fruition (Ricci et al. 2015). A lot of works have been developed in the last decade for recommending the most suitable items by applications in $\mathrm{CH}$ domain, especially considering several kinds of user information and different items' features (Amato et al. 2017).

A context-aware recommender system SMARTMUSEUM using ontology to manage heterogeneous CH's data and to support information needs of tourists has been proposed by Ruotsalo et al. (2013). They presented an ontology-based personalisation, annotation and information filtering framework that consists of vector space model-based 
retrieval method, query expansion, feature balancing and result clustering. A laboratory experiment with around 30 user profiles created and $500 \mathrm{CH}$ objects and a user trial with 24 participant and 25 subjects were conducted to show effectiveness of their framework for applying to RSs. Bartolini et al. (2013) introduced a general multimedia RS that able to uniformly manage heterogeneous multimedia data of $\mathbf{C H}$ objects and proposed context-aware recommendation techniques supporting intelligent multimedia services for users. On a separate way with low and high level information of the $\mathrm{CH}$ multimedia, they analysed the system which implicitly determines information about users and related communities. As an outdoor scenario, their application was evaluated with 10 users for 2 days by using Task Load Index (TLX) factor to validate its effectiveness of improving user experience. Then, Bartolini et al. (2016) extended the system by adding the recommendation function of dynamic visiting paths. Based on an indoor scenario at Capodimonte Museum in which 50 users participated for some weeks, the authors evaluated their system by comparing with User based Pearson Correlation (UPCC) and Item based Pearson Correlation (IPCC) to show the system's effectiveness for user satisfaction. In addition, Amato et al. (2017) proposed an extended version of the system using hyper-graph to model the social network of entire multimedia related to $\mathrm{CH}$ objects. The system was assessed by two experiments with around 50 participants to various complexity levels of tasks, in terms of TLX, the number of clicking mouse and time consumption. Smirnov et al. (2017) developed a tourist assistant application (TAIS) based on collaborative filtering technique and ontology-based context for supporting tourists in a $\mathrm{CH}$ region. It consists of a set of services joined together by a smart space that provides possibilities to organize semantic-based information exchanges between these services. The application was evaluated by analysing transactions of 1000 downloaders who acquire information of CH objects. Rajaonarivo et al. (2019) proposed a framework to recommend a sequence of $\mathrm{CH}$ Points Of Interests (POIs) to be visited by tourists. It integrates $\mathrm{CH}$ databases from heterogeneous sources by a unified data model following three standards in $\mathrm{CH}$ research community in France. Based the data model, a hybrid approach that benefits from content-based and collaborative filtering techniques recommends POIs related to $\mathrm{CH}$ objects. By a virtual experimentation with a sample of 120 POIs, the authors evaluated their framework in terms of average time and preference scores. Su et al. (2019) suggested a big data architecture to support typical $\mathrm{CH}$ recommendation applications. It relies on a context-aware hybrid recommendation strategy that is deployed on a multi-layer architecture based on big data and edge computing technologies, in order to dynamically provide sequences of POIs. With 50 users who installed an Android application (i.e., Smart Search Museum), the architecture was assessed in terms of TLX factors, and the authors compared their recommendation with UPCC and IPCC methods provided by Apache Mahout framework.

As afore-reviewed, there are many efforts to apply the RSs into CH domain. However, evaluations in their works are in a laboratory level or even based on real-world scenarios with small numbers of participants, because it is difficult to collect rating scores of users to $\mathrm{CH}$ objects. Furthermore, judging a visitors' preference based on the scores to $\mathrm{CH}$ objects would be careful or impossible due to their inestimable values. In addition to these, although context-aware RSs seem to like quite suitable to $\mathrm{CH}$ domain as most of the works have focused on the recommendation technique, tour preparation can be a fundamental step to an overall pleasant experience (Cardoso et al. 2018). In other words, there is potential to improve visitors' satisfaction by recommending items in the preparation step if we can use datasets including rating scores. Thus, we leverage dataset about restaurant facilities, which are closely related to a tourism for $\mathrm{CH}$ domain, to analyse cultural differences for RSs. Moreover, two methodologies are discussed for applying the results into the domain.

\subsection{Cross-cultural studies for recommender systems}

Cultural differences have been perceived as significant in tourism research, since visitors' cultural background is related to the experience they seek (Jung et al. 2018). The tourism industry combined with IT is becoming more and more international with increasing number of inbound tourists from different cultures (Li 2014). According to findings of Chu and Huang (2017), travellers from different cultural areas (and countries) have various rating behaviours. In addition, there are several researches to analyse the influences of cultural differences in RSs (Tang et al. 2011; Chen and Pu 2014; Choi et al. 2014; Berkovsky et al. 2018). These studies support an argument that RSs for $\mathrm{CH}$ domain should consider the cultural differences for visitors' higher satisfaction and enjoyment.

Chen and Pu (2008) evaluated cross-cultural difference by comparing an organisation-based product and a normal list view for recommended items, in terms of recommender interface. They proposed the organisation-based product recommendation following five design principles which had been presented by testing 13 interface prototypes by $\mathrm{Pu}$ and Chen (2006). The proposed view categorises recommended products and uses the category title as the explanation of multiple products. A survey with 120 volunteers from five countries (i.e., China, Swiss, France, Italy and Germany) was conducted for the two interfaces. And its results support that people from different cultures, especially between oriental and western cultures, have vary behaviours in the proposed view interface. Then, Chen and Pu (2014) extended 
the interface by considering users' preference elicited through a user interface. It is based on an association rule mining to produce dynamic and diverse critiques. Additionally the interface reflects users' current preferences and potential needs. The authors conducted various experiments using an eye-tracker and TLX factor, and one of the results revealed that there is significant preference gap between oriental and western participants to recommendation interface, even with similar effort needed. Tang et al. (2011) proposed a collaborative filtering-based multi-domain recommendation between films, TV series, books, games and musics. It performs a series of studies to reveal cultural influences to user preferences. Experiments with 333 students from Hong Kong and China for around 108 items showed that difference preferences to various cross-domain recommendations are caused by different cultures. Choi et al. (2014) analysed factors that influence user attitudes towards mobile RSs and examined how the factors interact with cultural values to affect the attitudes towards the RSs. They focused on collectivism and uncertainty avoidance (UA) as the two dimensions that are closely related to the functionality and purposes of mobile RSs. An on-line survey with 461 participants from three countries (i.e., Korea, China and UK) about the theoretical mobile RS using various data (i.e., users' previous App purchases, use patterns, etc.) was conducted. One of the survey results revealed that the both dimensions are differently affected by cultural differences. Berkovsky et al. (2018) examined user perceptions of nine trust factors to presentation, explanation and priority of recommended items by cultural differences. Their experiments with 102 subjects from four countries (i.e., France, Japan, Russia and USA) showed that user preference to the presentation and explanation methods is influenced by cultural difference. Chu and Huang (2017) extended a large-scale hotel information, which was collected from the Tripadvisor, to include heterogeneous hotel information and to facilitate multi-modal and cross-cultural analysis. They analysed rating scores of users from eight countries to various detailed features of hotels such as business service, check-in, front desk, cleanliness, overall, location, rooms, service, sleep quality and value. As a result, significant difference in rating behaviours for the hotel features was revealed. Furthermore, they predicted users' rating scores to hotels based on an extended MF by combining with various factors such as date, price, nationality, comment and the visual concept which is classified by support vector machine. To avoid misunderstanding about valuable contributions of researchers mentioned, we clearly say that we have only reviewed their results related to analysis of cultural differences and factors, because the others are out of scope for this paper.

Regarding above works, most of existing researches have analysed cultural differences along with the small number of countries, as Choi et al. (2014) mentioned one limitation in their paper. Whereas, our experiments consider around 80 countries. In addition, we analyse user preferences in terms of cross-cultural difference and discuss two methodologies to utilise the cross-cultural factors into RSs. Furthermore, the proposed model and its results are able to be used by general RSs, since we analyse the cultural differences by using computational techniques. In other words, all the results are numerical data that can be easily applied to other RSs.

\section{Computational analysis of cross-cultural factors}

\subsection{Definition of cross-cultural contextualisation}

In this section, we define a novel concept of Cross-Cultural Contextualisation (CCC) based on relevant definitions in the cross-cultural discipline to understand properly use of cultural difference in the field of RS. We start with the culture definition of Hofstede (1980) introduced in Sect. 1, as fundamental basis of our definition. He defined the term "culture" as "the collective programming of the mind which distinguishes the members of one human group from another". By inspired this definition, we recognised twofold features about cross-culture factors for RSs as follows:

- one is that collaborative filtering techniques are suitable to embrace cultural factors, as discussed by Choi et al. (2014) and as shown in Sect. 4.3.

- Another is that countries could be considered instead of individual user as elements in RSs, as shown in the analysis results reviewed in Sect. 2 and our analysis of dataset in Sect. 4.1

Heimbürger and Kiyoki (2014) defined several relevant concepts to CCC, as shown in Table 1. On the other hand, Park et al. (2018) defined "contextualisation" as "a process of drawing a meaningful results through a computational analysis to observable and obtainable resources". With referring these concepts, we define "cross-cultural contextualisation" for RSs as follows:

"a process of deriving and contextualising meaningful and useful cross-cultural factors between objects (i.e., users and items) by the computing technique(s) such as models, methods, functions or algorithms from crosscultural context in physical, virtual or hybrid recommendation environments".

In the next section, we present a contextualisation model based on MF technique regarding to this definition and explain relevant features. 
Table 1 Concepts and its definitions related to CCC (Heimbürger and Kiyoki 2014)

\begin{tabular}{ll}
\hline Concept & Definition \\
\hline Cross-cultural & $\begin{array}{c}\text { Concerns comparative studies and knowledge between two cultures. In case of several cultures we talk about } \\
\text { multi-cultural studies } \\
\text { Cross-cultural communication }\end{array}$ \\
$\begin{array}{l}\text { Consists of human-to-human, human-to-machine, and human-to-environment communication in cross-cultural } \\
\text { environments. The environment can be physical, virtual or hybrid } \\
\text { Research, development, design and implementation of computational models, methods, functions and algorithms } \\
\text { for cultural applications } \\
\text { Context } \\
\text { Context computing } \\
\text { Context computing can be defined as the use of context in software applications, where the applications adapt } \\
\text { to discovered contexts by changing their behaviour. A context-sensitive application presents the following } \\
\text { features: context sensing, presentation of information and services to a user, automatic execution of a service, } \\
\text { and tagging of context to information for later retrieval }\end{array}$
\end{tabular}

\subsection{Contextualisation of cross-cultural factors}

\subsubsection{Problem definition}

First, we use a scenario of the restaurant recommendation in the field of tourism to define a problem and objective considered in this paper. We consider users in general RSs as user groups per each country as will be discussed in Sect. 4.3. Thus, a vector can be referred as a set of user groups' preferences (i.e., rating scores) to each item (i.e., restaurant) and is expressed by:

$\boldsymbol{V}_{\boldsymbol{c}_{n}}=\left\langle f\left(c_{n}, r_{1}\right), \cdots, f\left(c_{n}, r_{M}\right)\right\rangle$,

where $f\left(c_{n}, r_{1}\right)$ indicates an average rating score of a specific culture (i.e., a user group or a country) $c_{n} \in C, n \in[1, N]$ to an arbitrary restaurant $r_{m} \in R, m \in[1, M]$. However, there might be biased rating scores due to some items being massively liked (Pavlidis 2019). Although several methods have been proposed such as significance weighting (Herlocker et al. 2017), default voting (Breese et al. 2013), inverse user frequency (Breese et al. 2013) and case amplification (Breese et al. 2013), we calculate average scores as a basic analysis for utilising cultural differences to RSs. The user and item biases will be handled in the next section.

Therefore, we are able to model cultural preferences by the following Cross-Cultural Contextualisation model as a observed rating matrix $\boldsymbol{A}$ regarding vectors $\boldsymbol{V}_{\boldsymbol{c}_{n}}$ for preference of $N$ cultures to $M$ items as

$A=\left[\begin{array}{c}V_{c_{1}} \\ \vdots \\ V_{c_{N}}\end{array}\right]=\left[\begin{array}{ccc}f\left(c_{1}, r_{1}\right) & \cdots & f\left(c_{1}, r_{M}\right) \\ \vdots & \ddots & \vdots \\ f\left(c_{N}, r_{1}\right) & \cdots & f\left(c_{N}, r_{M}\right)\end{array}\right]$.

However, what is critical in the matrix model as a collaborative filtering family of methods is the fact that it is generally sparse due to reflecting the lack of complete knowledge regarding the rating of items (Pavlidis 2019; Hong and Jung
2018). Therefore, we preprocess dataset used and utilise MF technique to interpolate the sparse matrix, as discussed in the next section. In this regard, our objective function is simply represented by

$\min \sum_{(n, m) \in(N, M)}\left(a_{n m}-\hat{a}_{n m}\right)$,

where the $a_{n m}$ and $\hat{a}_{n m}$ represent elements of the rating matrix $\boldsymbol{A}$ and an approximate matrix $\hat{\boldsymbol{A}}$, respectively.

\subsubsection{Cross-cultural contextualisation by matrix factorisation}

This section describes the process of utilising MF technique in order to predict missing preferences in the proposed contextualisation model. Note that our work is independent of the recommendation task, focussing primarily on the crosscultural analysis using the recommendation technique to handle cultural preferences to items. With respect to the above problem definition, the technique maps both cultures and items (i.e., restaurant) to a joint latent factor space of dimensionality $K$. The model (i.e., observed matrix) $\boldsymbol{A}$ can be represented by production of two matrices $\boldsymbol{P} \in \mathbb{R}^{N \times K}$ and $\boldsymbol{Q} \in \mathbb{R}^{K \times M}$ as follows:

$\left[\begin{array}{ccc}a_{11} & \cdots & a_{1 M} \\ \vdots & \ddots & \vdots \\ a_{N 1} & \cdots & a_{N M}\end{array}\right] \cong\left[\begin{array}{ccc}p_{11} & \cdots & p_{1 K} \\ \vdots & \ddots & \vdots \\ p_{N 1} & \cdots & p_{N K}\end{array}\right] \cdot\left[\begin{array}{ccc}q_{11} & \cdots & q_{1 M} \\ \vdots & \ddots & \vdots \\ q_{K 1} & \cdots & q_{K M}\end{array}\right]$,

where latent matrices $\boldsymbol{P}$ and $\boldsymbol{Q}$ are composed of characteristics vectors from $N$ cultures (i.e., countries) and $M$ items (i.e., restaurants), respectively. Each country $n$ is associated with a vector $\boldsymbol{p}_{n}$ and each restaurant $m$ is associated with a vector $\boldsymbol{q}_{\boldsymbol{m}}$. The elements of $\boldsymbol{p}_{\boldsymbol{n}}$ for a country $n$ indicate the interest degree of the country to restaurants that are high on the corresponding factors, while the elements of $\boldsymbol{q}_{\boldsymbol{m}}$ for a restaurant $m$ mean the interest degree of countries to which 
the restaurant possesses those factors. Thus, the dot product $p_{n}^{T} q_{m}$ captures the interaction between $n$th country and $m$ th restaurant, and an approximation $\hat{a}_{n m}$ of rating score of the country for the restaurant is estimated by

$a_{n m} \cong \hat{a}_{n m}=p_{n}^{T} q_{m}$.

Singular Value Decomposition (SVD) is a low-rank decomposition method designed to obtain latent factors. It disassembles the observed matrix $\boldsymbol{A}$ into the two characteristic matrices of country and restaurant. In this regard, we need to minimise errors between actual scores $a_{n m}$ in the observed matrix $\boldsymbol{A}$ and predicted scores of Equation 5, and minimising the error can be formulated as sum-square distance based on the renewal rule of gradient descent by:

$\min \sum_{(n, m) \in \Gamma}\left(a_{n m}-\sum_{k=1}^{K} p_{n k} q_{k m}\right)^{2}+\lambda\left(\left\|p_{n}\right\|^{2}+\left\|q_{m}\right\|^{2}\right)$,

where, $\Gamma$ is the set of $(n, m)$ pairs for which $a_{n m}$ is known (i.e., training set), and the constant $\lambda$ controls regularization terms to avoid over-fitting problem. Note that $\|\cdot\|^{2}$ is the $L^{2}$ -norm in which $\|x\|^{2}=\left(\sum_{k} x_{k}^{2}\right)^{1 / 2}$ with $k$ being the dimensions or components of $x$.

As discussed in Sect. 3.1, to avoid user and item biases, we introduce the baseline as follows:

$b_{n m}=\mu+b_{n}+b_{m}$,

where $b_{m m}$ indicates the basis value for predicting relationship between country $n$ and restaurant $m$, and $b_{n}$ is the difference between an average score given by the country to all restaurants and an average of the overall ratings, and $b_{m}$ is the difference between rating scores given to the restaurant by all the countries and an overall rating to the restaurant, respectively. The $\mu$ is an average of overall ratings. In other words, $b_{n}$ and $b_{m}$ reflect the deflection values observed in the average rating of the country and the restaurant. By applying this, the Equation 6 is able to be expressed by

$$
\begin{aligned}
& \min \sum_{(n, m) \in \Gamma}\left(a_{n m}-b_{n m}-\sum_{k=1}^{K} p_{n k} q_{k m}\right)^{2} \\
& \quad+\lambda\left(b_{n m}^{2}\left\|p_{n}\right\|^{2}+\left\|q_{m}\right\|^{2}\right) .
\end{aligned}
$$

Since Eq. (8) is a convex problem, it is possible to optimize it using the gradient descent.
Table 2 Summary of dataset used in references

\begin{tabular}{llll}
\hline References & \# of items & \# of users & $\begin{array}{l}\text { \# of } \\
\text { coun- } \\
\text { tries }\end{array}$ \\
\hline $\begin{array}{l}\text { Chen and Pu (2008) } \\
\text { and Chen and Pu }\end{array}$ & $Q$ & 120 & 5 \\
$\begin{array}{l}\text { (2014) } \\
\text { Tang et al. (2011) }\end{array}$ & 108 & 333 & \\
$\begin{array}{l}\text { Choi et al. (2014) } \\
\text { Chu and Huang (2017) }\end{array}$ & $Q$ & Unk. (i.a. 12,773) & Unk. (i.a. 1.3M) \\
Choi et al. (2014) & $Q$ & 461 & 8 \\
\hline
\end{tabular}

The $\mathrm{Q}$ and Unk. indicate the questionnaire use (not using rating score) and the unknown, respectively. Note that we only mentioned dataset used for cross-cultural analysis among evaluations

\section{Evaluation}

\subsection{Dataset}

In this section, we explain statistical information of dataset used and reasons of why we use the dataset for cross-cultural analysis with referring other datasets that have been used in studies reviewed in Sect. 2. As shown in Table 2, more than half of related works ( $3 / 5)$ didn't use rating scores of users to items in order to analyse cultural difference. Even though the two other researches used rating scores for their recommendation, they considered the small number of countries (i.e., different cultures).

We used the dataset of restaurant review downloaded from the link ${ }^{1}$ of Kaggle, since to the best of our knowledge, there was no dataset which has users' rating scores to $\mathrm{CH}$ objects. As will be discussed in Sect. 5, we are able to link this data to recommending POIs related to $\mathrm{CH}$ objects by considering cross-domain recommendation. The dataset includes travellers' reviews to restaurants at London in UK collected from Tripadvisor whose traffic flow continues to show an upward tendency and it becomes one of the top 250 websites in the world, according to Alexa traffic statistics ${ }^{2}$. In the on-line service, users share their experience to hotels and restaurants.

The dataset contains 19,999 reviews of 15,424 users to 2413 restaurants and also includes user's rating scores, visit date, approximate locations of the user and restaurant. Although there are four kinds of rating score for overall, food, value and service, we only considered the overall rating scores because of the lack of other scores. Therefore,

\footnotetext{
1 https://www.kaggle.com/residentmario/exploring-tripadvisor-ukrestaurant-reviews

${ }^{2}$ http://www.alexa.com/siteinfo/tripadvisor.com, Retrieved: July 9th, 2019
} 
Table 3 Statistic of dataset used

\begin{tabular}{ll}
\hline Pro. name & Value \\
\hline \# of restaurants & 50 \\
\# of countries & 81 \\
\# of reviews & 11,280 \\
Missing value & 487 \\
Sparsity (\%) & $12.025 \%$ \\
\hline
\end{tabular}

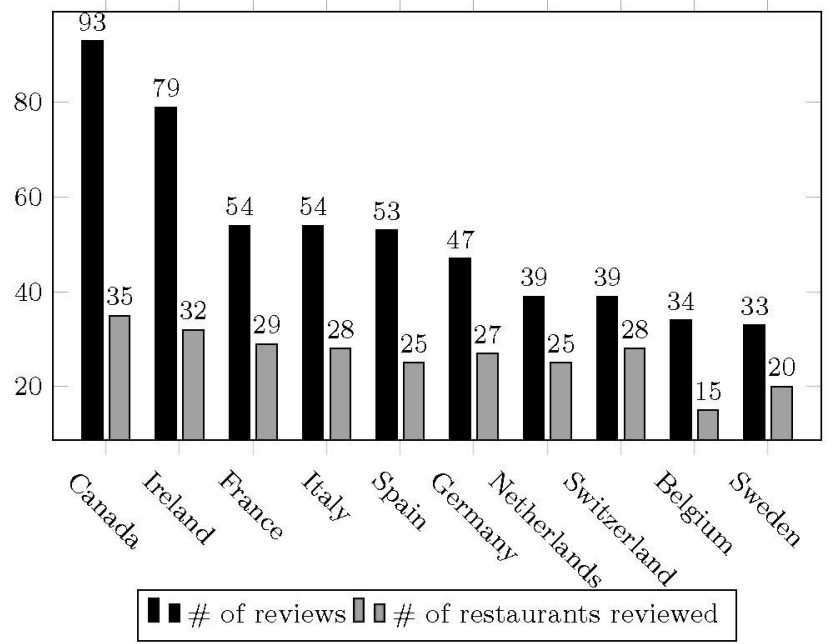

Fig. 1 Number of reviews and restaurants of top 10 countries

sparsity of the dataset, i.e., the number of zero-valued elements (i.e., missing values) divided by the total number of elements in a matrix, is very high as $0.0537 \%$. In real-world situations, this high sparsity is natural but hampers the recommendation process (Pavlidis 2019). Since our purpose is to contextualise cross-cultural difference, we carefully consider the sparsity affecting the prediction accuracy of the proposed method consisting of country-wise vectors. Therefore, we filtered items which have given few rating by users and grouped users into countries. Finally, we have 11,280 average rating scores of 81 countries to 50 restaurants as shown in Table 3.

To see detailed information of the dataset, we rank countries in terms of their review numbers. Figure 1 shows the numbers of review and restaurant of top ten countries. To improve the readability of the figure, we excluded top three countries (i.e., UK, USA and Australia), since they have much more reviews than the others. UK has around 6,000 reviews ( 49 restaurants), USA has around 740 reviews (45 restaurants) and Australia has around 200 reviews (41 restaurants), respectively. Most of top ranked countries are in Europe. It is common phenomenon, since all restaurants in the dataset is located at London in UK. Additionally, we summary 81 countries in Table 4 . In terms of classification
Table 4 Country distribution by the number of reviews

\begin{tabular}{lllll}
\hline & Over 100 & $40-99$ & $20-39$ & $5-19$ \\
\hline \# of country & 3 & 6 & 28 & 44 \\
Aver. \# of review & 2673 & 63 & 29 & 11 \\
$\begin{array}{c}\text { Aver. \# of restau- } \\
\text { rants reviewed }\end{array}$ & 45.000 & 29.333 & 15.285 & 3.090 \\
$\begin{array}{c}\text { Continents } \\
\text { Ty }\end{array}$ & EU, NA, AU & EU, NA, SA, AU & All & AS, SA
\end{tabular}

We except for Antarctica, since there are no countries. Africa: AF, Asia: AS, Europe: EU, North America: NA, South America: SA, Australia: AU

by seven continents ${ }^{3}$ (except for Antarctica), the used dataset seems have various cultures in geographical aspect. For the average numbers of review and restaurant, it is able to be said that the dataset has a large enough number of reviews per each country for cross-cultural analysis through MF technique.

\subsection{Measurements}

We employ three measurements to evaluate rating prediction performance of the CCC model based on MF and to analyse cross-cultural difference through k-mean clustering techniques. Comparing predicted ratings with ground truths, we calculate prediction error based on Root Mean Square Error (RMSE) by

$R M S E=\sqrt{\sum_{n, m}\left(a_{n m}-\hat{a}_{n m}\right)^{2} / N_{t}}$,

where $\hat{a}_{n m}$ is the predicted value of the rating that the country $n \in N$ rates item $m \in M, a_{n m}$ is the ground truth and $N_{t}$ is the total number of test ratings.

In order to measure cultural differences, we calculate distances between countries based on angular and Euclidean distances. The former is obtained based on the cosine similarity which is formulated as

$S^{C}\left(c_{i}, c_{j}\right)=\frac{V_{c_{i}} \cdot V_{c_{j}}}{\left\|V_{c_{i}}\right\|\left\|V_{c_{j}}\right\|}$,

where $c_{i}$ and $c_{j}$ indicate two cultures (i.e., countries). The $\boldsymbol{V}_{\boldsymbol{c}_{i}}$ and $\boldsymbol{V}_{c_{j}}$ are from Eq. (1). We use an inverse function of cosine to convert it into the angular distance by

$D^{A}\left(c_{i}, c_{j}\right)=\frac{1}{\pi} \times 2 \cos ^{-1}\left(S^{C}\left(c_{i}, c_{j}\right)\right)$.

And then it is combined with Euclidean distance $D^{E}\left(c_{i}, c_{j}\right)=\left\|V_{c_{i}}-V_{c_{j}}\right\|=\sqrt{\left(V_{c_{i}}-V_{c_{j}}\right) \cdot\left(V_{c_{j}}-V_{c_{i}}\right)}$ to cal-

\footnotetext{
${ }^{3}$ https://en.wikipedia.org/wiki/Continent
} 


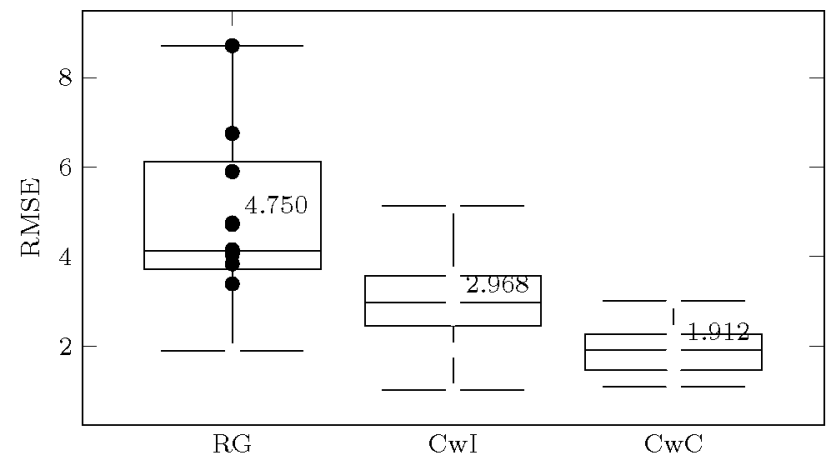

Fig. 2 Rating prediction performance by RMSE

Table 5 Detailed performance results

\begin{tabular}{llll}
\hline & RG & CwI & CwC \\
\hline Average value & 4.750 & 2.968 & 1.912 \\
Standard deviation & 1.918 & 1.089 & 0.570 \\
Median value & 4.123 & 2.973 & 1.916 \\
\hline
\end{tabular}

culate cultural difference $D_{i j}^{C}$ between two cultures $c_{i}$ and $c_{j}$ by

$D_{i j}^{C}=D^{A}\left(c_{i}, c_{j}\right) \cdot D^{E}\left(c_{i}, c_{j}\right)$.

\subsection{Analysis of cross-cultural contextualisation}

We conducted ten-fold cross-validation scheme to measure the accuracy of predicted rating matrix by RMSE. In order to validate the effectiveness of the preprocessing for matrix sparsity, we compared two predicted matrices by the proposed model (i.e., using MF) for original data of individuals, called $\mathrm{CwI}$, and filtered data for cultures, named $\mathrm{CwC}$, and a matrix filled to missing elements by random values, called RG. Figure 2 shows the rating prediction performance (i.e., RMSE) of these three methods. As shown in the figure, using the proposed method based on MF technique helps to improve the accuracy in terms of RMSE than generating random values. Also, standard deviations of $\mathrm{CwI}$ and $\mathrm{CwC}$ are more smaller than that of RG. In other words, the $\mathrm{CwI}$ and $\mathrm{CwC}$ are more stable than the $\mathrm{RG}$, as listed in Table 5. In addition, comparing between $\mathrm{CwI}$ and $\mathrm{CwC}$, the $\mathrm{CwC}$ outperforms the $\mathrm{CwI}$. It supports that analysing rating matrix per country-wise is a right way for cross-cultural analysis, than considering rating matrix consisting of individuals' vectors. In the next section, countries are clustered based on the predicted culture vector $V_{c_{n}}$ to know whether there are distinguishable cultural areas on the rating behaviours of travellers to restaurants or not.

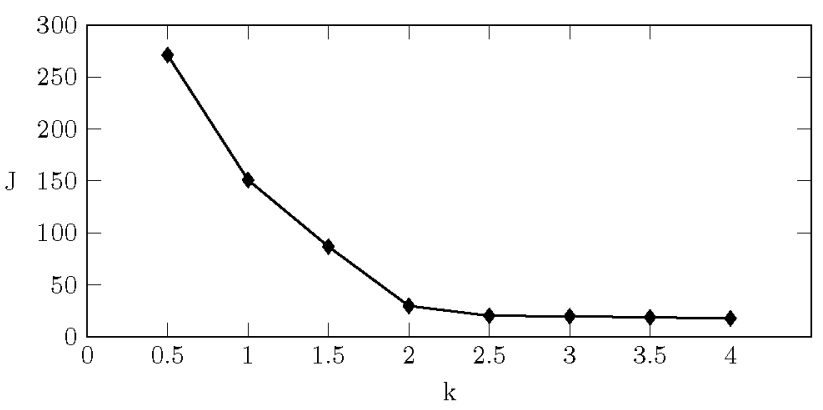

Fig. 3 Elbow method for optimal k

\subsection{Analysis of cross-cultural difference}

To analyse cross-cultural differences, we utilise the $k$-means clustering technique which is well-known. It groups items based on an intra-compactness among items within each cluster. Therefore, given a set of observation (i.e., countries $\left.\left(c_{1}, c_{2}, \ldots, c_{n}\right)\right)$, where each observation is a $d$-dimensional real vector (i.e., the number of restaurants, $d=m$ ), $k$-means clustering aims to partition the $n$ observations into $k(\leq n)$ sets $S=\left\{S_{1}, S_{2}, \ldots, S_{k}\right\}$, so as to minimise the intra-compactness. Thus, the objective is formally to find

$\arg \min _{S} \sum_{i=1}^{k} \sum_{c \in S_{i}}\left\|c-\mu_{i}\right\|^{2}$,

where $\mu_{i}$ is the mean of points in $S_{i}$. This is equivalent to minimizing the pairwise squared deviations of points in the same cluster. Thus, it can be converted by using Equation 12 as follows:

$J=\underset{S}{\arg \min } \sum_{i=1}^{k} \sum_{c_{n} \neq c_{m} \in S_{i}} D_{m m}^{C}$.

Through an iterative refinement including expectation and maximisation steps, the algorithm finds a proper $k^{\prime}$, which satisfies the above objective function, from 1 to arbitrary $k$. As shown in Fig. 3, the more the number $k$ of cluster increases, the more the sum of distance J steeply decreases, before $k$ is 2. If the plot looks like an arm, then the elbow on the arm is optimal (i.e., $k=2$ ). From the results of clustering countries, we found some interesting outcomes as follows:

1. Most of countries were classified into two Western and Eastern cultural areas if we group them into two clusters, as other researchers have found in their works (Chen and Pu 2008; Tang et al. 2011; Chen and Pu 2014).

2. When countries are grouped into more delicate areas (i.e., k=3), Hong Kong, Philippines, Vietnam and so on belonged to another cultural area. The reason may be that they have been affected by English-speaking country in the past. 


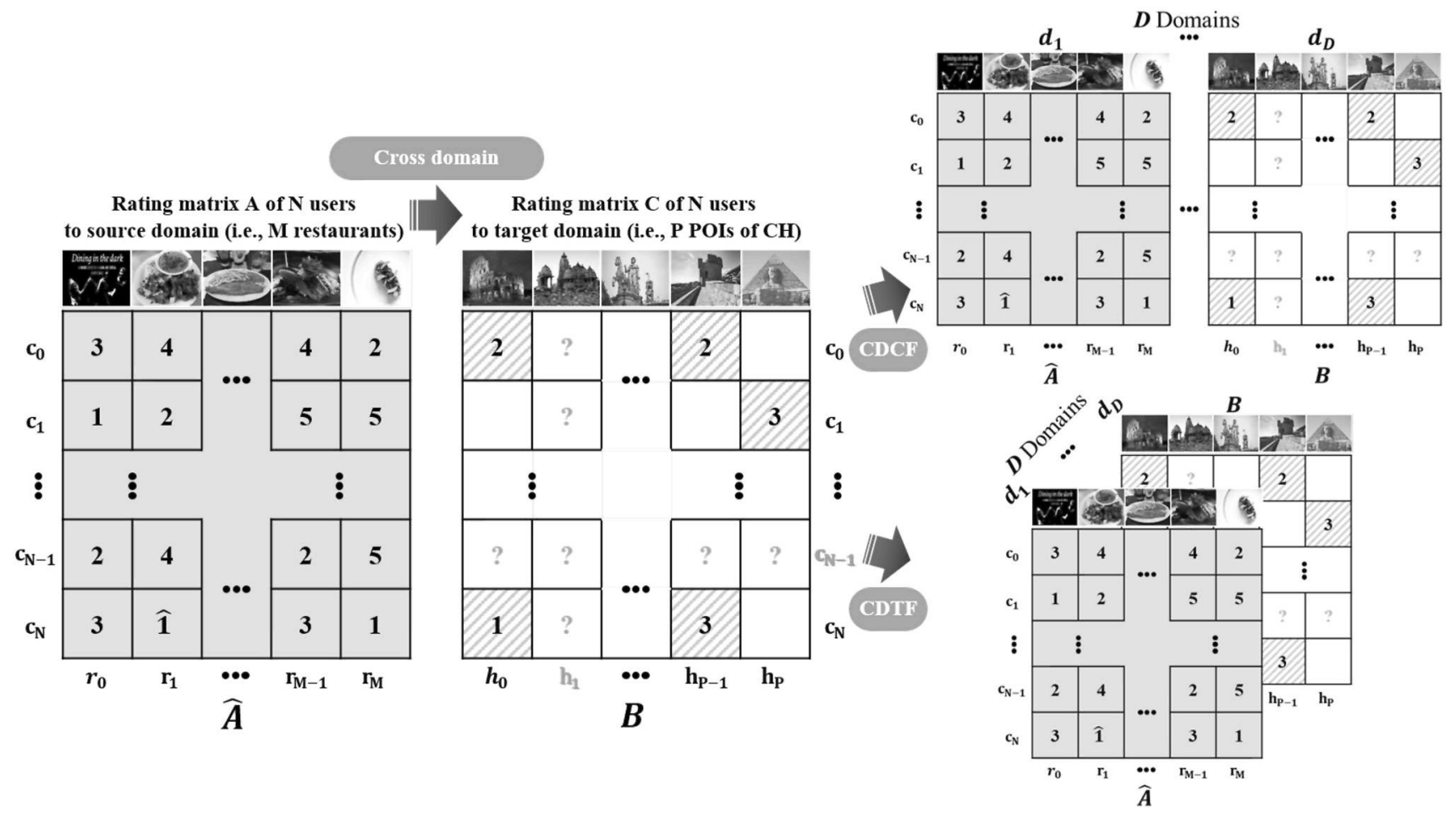

Fig. 4 Overview of cross-domain recommendation using CCC model

3. In the cluster of Eastern area, users from Korea and Japan have much more similar each other, than with China. It seems that China has a huge territory and then has developed various sub-cultures, by comparing with Korea and Japan. These two countries might have similar preferences for food culture because of the relatively smaller area surrounded by sea.

4. Except for these results, there were several interesting issues, however we were not able to figure out the reasons. It implies that our results should be analysed along with researchers in the field of humanities disciplines to find more valuable meanings.

We discussed findings obtained from the above analysis of the cross-cultural difference by using the computational method based on MF and $k$-means clustering techniques. In the next section, we discuss methodologies, inspired by other researchers' contributions, to utilise the proposed model to recommendation in $\mathrm{CH}$ domain.

\section{Discussion}

This section introduces two methodologies in order to utilise the proposed model to $\mathrm{CH}$ domain for recommending POIs. We briefly discuss from general issues in RSs to cross-domain recommendation before presenting the two methodologies. According to Pavlidis (2019), the most important challenges are the sparsity and the cold-start problem. The high sparsity is a very commoncondition in the real-world, especially when the number of items are much more than that of the users which hampers the ability of the system to make accurate predictions ( $\mathrm{Su}$ and Khoshgoftaar 2009). The cold-start problem leads to the inability or difficulty of providing accurate recommendations for new items and new users (Schein et al. 2002). Cross-domain recommendation hence is becoming an emerging research topic in the field of RSs.

Figure 4 draws the overview of how to apply the proposed model to cross-domain recommendation. The matrix $\hat{\boldsymbol{A}}$ is the approximate one from the proposed model for recommending restaurants. Whereas, the matrix $\boldsymbol{B}$ indicates a rating matrix for the POIs related to $\mathrm{CH}$ objects and might be really sparse with new items and users, as discussed in Sect. 4.1. Gray colour indicates approximate scores in the matrix $\hat{\boldsymbol{A}}$, and diagonal line in the matrix $\boldsymbol{B}$ expresses observed rating scores. For simplify, we express positive integers as estimated and observed rating scores in the matrices. Assume that there are new users $c_{N-1}$ or points $h_{P-1}$ for the $\mathrm{CH}$ domain. Since the proposed model is capable of fully filling in the rating matrix $\boldsymbol{A}$ into $\hat{A}$ thank to MF technique, the relatively abundant model (i.e., $\hat{\boldsymbol{A}}$ ) than a normal matrix is able to improve prediction accuracy of missing values in the sparse matrix $\boldsymbol{B}$ which might have new users and items. 
To technically deal with the sparsity and cold-start problems, we introduce two approaches named as Cross Domain Collaborative Filtering (CDCF) of Li (2011) and Crossdomain Triadic Factorization (CDTF) of Hu et al. (2013), as shown in Fig. 4. The former is straightforward by performing MF to a horizontal concatenation of matrices for all domains (i.e., restaurant and POI in $\mathrm{CH}$ ). For the details, refer to Li (2011). For the method, given $D=\left\{D_{1}^{i}, D_{2}^{i}, \ldots, D_{D}^{i}\right\}$ domains for $U$ users and to $I, J, \ldots, D$ items per domain, time complexity is $\mathcal{O}(U \times(I+J+\cdots+L))$. The latter used CP model, which is an extension of PARAFAC2, as one of tensor factorisation techniques. Therefore, for the same condition with the former one, time complexity is $\mathcal{O}(U \times R \times D \times R))$, where the $R$ indicates the number of virtual items. Even though it seems much more heavy with respect to resource needs, the CDTF is better in terms of accuracy, according to $\mathrm{Hu}$ et al. (2013). For the details, read to Hu et al. (2013).

However as found by Tang et al. (2011), the more domains used are close each other, the performance of crossdomain recommendation is better. Thus, domains for the recommendation should be carefully considered. As another way, weights, which present correlations between domains, could be reflected to improve the accuracy of recommendation performance. The weight is able to be obtained by using correlation analysis such as Pearson correlation coefficient.

\section{Conclusion}

$\mathrm{CH}$ domain as places where people interact with objects capable of arousing interest and excitement is significantly shifting from traditional heritage sites into smart cultural heritage environment through vary technologies such as RS, Internet of Things, WSN, VR/AR and 5G. RSs, as one important technology in the smart space, have been widely used to personalised services and applications. Whereas, cultural difference is often considered a barrier to technology adoption. However, few works focus on how cultural difference affect recommendation despite the factor largely influences user rating behaviours to the RSs. Moreover, existing studies have mostly focused on analysis of evaluation results of their recommendation to reveal cultural differences, rather than utilisation of the cross-cultural factors into RSs.

Therefore in this paper, we defined a novel concept of cross-cultural contextualisation regarding relevant concepts such as culture, cross-cultural, cultural computing and context. Regarding the definition, a model based on MF and clustering techniques was proposed to compute the crosscultural factor affecting users (countries or cultures) preferences. In addition, we discussed two methodologies to apply the model into RSs in $\mathrm{CH}$ domain through cross-domain recommendation techniques. Experiments results with a real-world dataset, which contains 11,280 reviews rated by various visitors from 81 countries to 50 restaurants, showed effectiveness of the proposed model and supported that there is cultural difference influencing user rating behaviours in recommendation. Furthermore, a systematic analysis of dataset and the experimental results presented that individual users could be considered as country-wise groups for recommendation in $\mathrm{CH}$ domain.

However, some limitations by dataset are still remained in our analysis. One is diversity deficiency of recommended items. In other words, restaurants considered as items are located in UK, especially London, because of the limitation of gathering datasets. Thus, it is difficult to avoid the data unbalance that there are much more reviews of people who live in UK than the others. It might have impacted our analysis. However, as discussed in Sect. 4.1, the systematic analysis of dataset showed that our preprocessing would have reduced the danger, since it leaded to high accuracy performance of our model, as shown in Sect. 4.4. Another is nationality uncertainty of reviewers. Since we were not able to directly obtain nationalities of reviewers by user privacy issue, even we had carefully checked their location, the country information might be incorrect in some cases. For example, a location information of one Korean, who is living in Norway, could be expressed one place in Norway.

Therefore, we plan to collect rating reviews to restaurant from various places and to find a solution to fix the uncertainty for country information. Furthermore, as discussed in Sect. 5, we will implement and evaluate a recommender system considering the proposed model to validate its effectiveness and users' satisfaction to recommendation in $\mathrm{CH}$ domain. In addition, our evaluations will be extended by using other clustering techniques, and the Elbow method would be compared with the others such as gap statistic, silhouette index, sum of squares method, in order to find a more proper way classifying cross-cultures. Moreover, we plan to introduce sentiment analysis in the CCC. Because the analysis has not been currently covered in the state of the art, especially $\mathrm{CH}$ recommendation, despite the importance of sentiment analysis. And the sentimental analysis will give a chance to address difficulty of measuring users' rating score to $\mathrm{CH}$.

Finally we emphasise that the computational techniques used were leveraged to computationally contextualise crosscultural factors and to analyse cultural differences between various cultures and countries which impact to user preferences, rather than to recommend items. Therefore, the proposed model and computing results capable of utilisation into the other RSs as well as various research fields.

Acknowledgements This research was supported by the Chung-Ang University Research Grants in 2018. Also, This work was supported by 
the National Research Foundation of Korea (NRF) grant funded by the Korea government (MSIP) (NRF-2017R1A2B4010774).

\section{References}

Amato F, Moscato V, Picariello A, Colace F, Santo MD, Schreiber FA, Tanca L (2017) Big data meets digital cultural heritage: design and implementation of scrabs, a smart context-aware browsing assistant for cultural environments. J Comput Cult Herit (JOCCH) 10(1):6

Bartolini I, Moscato V, Pensa RG, Penta A, Picariello A, Sansone C, Sapino ML (2013) Recommending multimedia objects in cultural heritage applications. In: Petrosino A, Maddalena L, Pala P (eds) Proceedings of the 2013 international workshops on new trends in image analysis and processing ICIAP 2013, vol 8158. Lecture notes in computer science. Springer, Naples, Italy, pp 257-267. https://doi.org/10.1007/978-3-642-41190-8_28

Bartolini I, Moscato V, Pensa RG, Penta A, Picariello A, Sansone C, Sapino ML (2016) Recommending multimedia visiting paths in cultural heritage applications. Multimedia Tools Appl 75(7):3813-3842. https://doi.org/10.1007/s11042-014-2062-7

Bekele MK, Pierdicca R, Frontoni E, Malinverni ES, Gain J (2018) A survey of augmented, virtual, and mixed reality for cultural heritage. JOCCH 11(2):7:1-7:36. https://doi.org/10.1145/3145534

Berkovsky S, Taib R, Hijikata Y, Braslavski P, Knijnenburg BP (2018) A cross-cultural analysis of trust in recommender systems. In: Mitrovic T, Zhang J, Chen L, Chin D (eds) Proceedings of the 26th conference on user modeling, adaptation and personalization, UMAP 2018, ACM, Singapore, pp 285-289. https://doi. org/10.1145/3209219.3209251

Breese JS, Heckerman D, Kadie CM (2013) Empirical analysis of predictive algorithms for collaborative filtering. CoRR. arXiv :abs/1301.7363

Cardoso PJS, Guerreiro PMM, Monteiro JM, Rodrigues JMF (2018) Applying an implicit recommender system in the preparation of visits to cultural heritage places. In: Antona M, Stephanidis C (eds) Proceedings of the 12th international conference on universal access in human-computer interaction (UAHCI 2018), vol 10908. Lecture notes in computer science. Springer, Las Vegas, NV, USA, pp 421-436. https://doi.org/10.1007/978-3-319-92052 $-8 \_33$

Chen L, Pu P (2008) A cross-cultural user evaluation of product recommender interfaces. In: Pu P, Bridge DG, Mobasher B, Ricci F (eds) Proceedings of the $2008 \mathrm{ACM}$ conference on recommender systems, RecSys 2008, ACM, Lausanne, Switzerland, pp 75-82. https://doi.org/10.1145/1454008.1454022

Chen L, Pu P (2014) Experiments on user experiences with recommender interfaces. Behav IT 33(4):372-394. https://doi. org/10.1080/0144929X.2012.719034

Chianese A, Piccialli F (2014) Smach: a framework for smart cultural heritage spaces. In: Yétongnon K, Dipanda A, Chbeir R (eds) Proceedings of the 10th international conference on signal-image technology and internet-based systems, SITIS 2014, IEEE Computer Society, Marrakech, Morocco, pp 477-484. https://doi. org/10.1109/SITIS.2014.16

Chianese A, Piccialli F (2016) A smart system to manage the context evolution in the cultural heritage domain. Comput Eleetr Eng 55:27-38. https://doi.org/10.1016/j.compeleceng.2016.02.008

Chianese A, Piccialli F, Valente I (2015) Smart environments and cultural heritage: a novel approach to create intelligent cultural spaces. J Locat Based Serv 9(3):209-234. https://doi. org/10.1080/17489725.2015.1099752

Chianese A, Piccialli F, Jung JE (2016) The internet of cultural things: towards a smart cultural heritage. In: Yétongnon $\mathrm{K}$,
Dipanda A, Chbeir R, Pietro GD, Gallo L (eds) Proceedings of the 12th international conference on signal-image technology $\&$ internet-based systems, SITIS 2016, IEEE Computer Society, Naples, Italy, pp 493-496. https://doi.org/10.1109/SITIS .2016 .83

Choi J, Lee HJ, Sajjad F, Lee H (2014) The influence of national culture on the attitude towards mobile recommender systems. Technol Forecast Soc Change 86:65-79

Chu W, Huang W (2017) Cultural difference and visual infor mation on hotel rating prediction. World Wide Web 20(4):595-619. https:// doi.org/10.1007/s11280-016-0404-2

Heimbürger A, Kiyoki Y (2014) Context and user-centered approaches: icons in cross-cultural context. In: Brézillon P, Gonzalez AJ (eds) Context in computing-a cross-disciplinary approach for modeling the real world. Springer, Berlin, pp 309-325. https://doi. org/10.1007/978-1-4939-1887-4_20

Herlocker JL, Konstan JA, Borchers A, Riedl J (2017) An algorithmic framework for performing collaborative filtering. SIGIR Forum 51(2):227-234. https://doi.org/10.1145/3130348.3130372

Hofstede G (1980) Culture and organizations. Int Stud Manag Organ $10(4): 15-41$

Hofstede G, Bond MH (1988) The confucius connection: from cultural roots to economic growth. Organ Dyn 16(4):5-21

Hong M, Jung JJ (2018) Multi-sided recommendation based on social tensor factorization. Inf Sci 447:140-156. https://doi. org/10.1016/j.ins.2018.03.019

Hong M, Jung JJ, Piccialli F, Chianese A (2017) Social recommendation service for cultural heritage. Pers Ubiquitous Comput 21(2):191-201. https://doi.org/10.1007/s00779-016-0985-x

Hu L, Cao J, Xu G, Cao L, Gu Z, Zhu C (2013) Personalized recommendation via cross-domain triadic factorization. In: Schwabe D, Almeida VAF, Glaser H, Baeza-Yates RA, Moon SB (eds) Proceedings of the 22 nd international world wide web conference, WWW '13, international world wide web conferences steering committee/ACM, Rio de Janeiro, Brazil, pp 595-606. https://doi. org/10.1145/2488388.2488441

Jung TH, Lee H, Chung N, tom Dieck MC (2018) Cross-cultural differences in adopting mobile augmented reality at cultural heritage tourism sites. Int J Contemp Hosp Manag 30(3):1621-1645

Lee LYS (2016) Hospitality industry web-based self-service technology adoption model: a cross-cultural perspective. J Hosp Tour Res 40(2): 162-197

Li B (2011) Cross-domain collaborative filtering: a brief survey. In: Proceedings of the IEEE 23rd international conference on tools with artificial intelligence, ICTAI 2011, IEEE Computer Society, Boca Raton, FL, USA, pp 1085-1086. https://doi.org/10.1109/ ICTAI.2011.184

Li M (2014) Cross-cultural tourist research: a meta-analysis. J Hosp Tour Res 38(1):40-77

Nguyen TT, Camacho D, Jung JE (2017) Identifying and ranking cultural heritage resources on geotagged social media for smart cultural tourism services. Pers Ubiquitous Comput 21(2):267-279. https://doi.org/10.1007/s00779-016-0992-y

Park J, Lee O, Jung JE (2018) Spatio-temporal query contextualization for microtext retrieval in social media. Concurr Comput Pract Exp 30(15):e4458. https://doi.org/10.1002/cpe.4458

Pavlidis G (2019) Recommender systems, cultural heritage applications, and the way forward. J Cult Herit 35:183-196

Pu P, Chen L (2006) Trust building with explanation interfaces. In: Paris C, Sidner CL (eds) Proceedings of the 11th international conference on intelligent user interfaces, IUI 2006, ACM, Sydney, Australia, pp 93-100. https://doi.org/10.1145/1111449.1111475

Rajaonarivo L, Fonteles A, Sallaberry C, Bessagnet MN, Roose P, Etcheverry P, Marquesuzaà C, Lacayrelle ALP, Cayèré C, Coudert Q (2019) Recommendation of heterogeneous cultural heritage objects for the promotion of tourism. ISPRS Int J Geo Inf 8(5):230 
Ricci F, Rokach L, Shapira B (2015) Recommender systems: introduction and challenges. In: Ricci F, Rokach L, Shapira B (eds) Recommender systems handbook. Springer, Berlin, pp 1-34. https ://doi.org/10.1007/978-1-4899-7637-6_1

Ruotsalo T, Haav K, Stoyanov A, Roche S, Fani E, Deliai R, Mäkelä E, Kauppinen T, Hyvönen E (2013) SMARTMUSEUM: a mobile recommender system for the web of data. J Web Semant 20:5067. https://doi.org/10.1016/j.websem.2013.03.001

Schein AI, Popescul A, Ungar LH, Pennock DM (2002) Methods and metrics for cold-start recommendations. In: Järvelin K, Beaulieu M, Baeza-Yates RA, Myaeng S (eds) Proceedings of the 25th annual international $\mathrm{ACM}$ SIGIR conference on research and development in information retrieval, SIGIR 2002, ACM, Tampere, Finland, pp 253-260. https://doi.org/10.1145/564376.56442 1

Siountri K, Skondras E, Vergados DD (2018) A delivery model for cultural heritage services in smart cities environments. In: Ioannides M, Fink EE, Brumana R, Patias P, Doulamis A, Martins J, Wallace $M$ (eds) Proceedings of the 7 th international conference on digital heritage. Progress in cultural heritage: documentation, preservation, and protection, EuroMed 2018, vol 11197. Lecture Notes in Computer Science, Springer, Nicosia, Cyprus, pp 279-288. https ://doi.org/10.1007/978-3-030-01765-1_31

Smirnov AV, Kashevnik AM, Ponomarev A (2017) Context-based infomobility system for cultural heritage recommendation: tourist
assistant-TAIS. Pers Ubiquitous Comput 21(2):297-311. https ://doi.org/10.1007/s00779-016-0990-0

Su X, Khoshgoftaar TM (2009) A survey of collaborative filtering techniques. Adv Artif Intell 2009:421425:1-421425:19. https:// doi.org/10.1155/2009/421425

Su X, Sperli G, Moscato V, Picariello A, Esposito C, Choi C (2019) An edge intelligence empowered recommender system enabling cultural heritage applications. IEEE Trans Ind Inf 15(7):4266-4275

Tang TY, Winoto P, Ye RZ (2011) Analysis of a multi-domain recommender system. In: Proceedings of the 3 rd international conference on data mining and intelligent information technology applications, IEEE, Macao, China, pp 280-285

Wang Y, Dai X, Jung JJ, Choi C (2018) Performance analysis of smart cultural heritage protection oriented wireless networks. Future Gen Comp Syst 81:593-600. https://doi.org/10.1016/j.futur e.2017.04.007

Publisher's Note Springer Nature remains neutral with regard to jurisdictional claims in published maps and institutional affiliations. 